\title{
The role of vortex wake dynamics in the flow-induced vibration of tube arrays
}

\author{
N. K.-R. Kevlahan \\ Department of Mathematics $\mathcal{E}$ Statistics, McMaster University, Hamilton ON L8S 4K1, Canada
}

\begin{abstract}
Potential flow and two-dimensional Navier-Stokes calculations are used to investigate the role of vortex shedding in the non-resonant flow-induced vibration of periodic tube arrays. This dual approach untangles the effects of potential and vortical flow. The negative damping theory is shown to be inconsistent with the Navier-Stokes simulations, and allowing only a single degree of freedom in tube motion significantly overestimates the critical velocity. In contrast, Navier-Stokes simulations which allow all tubes to move in both the transverse and streamwise directions give results in good agreement with experiment. Somewhat surprisingly, potential flow calculations including an artificial phase lag between fluid force and tube motion give reasonably accurate results for a wide range of phase lags. This may be due to the fact that the most unstable mode at onset appears to be streamwise anti-phase (not whirling), as observed in the potential flow case.
\end{abstract}

Keywords: 76F65, 74F10, 76B47

\section{Introduction}

The role of vortex wake dynamics in the flow-induced vibration of isolated freely oscillating cylinders is now quite well understood (Williamson and Govardhan, 2004). In particular, Williamson and Govardhan (2004) discovered that for low mass damping ratios there exists a critical mass ratio below which the regime of high amplitude oscillations extends to infinite velocities. They also found that as velocity increases at fixed (small) mass damping there is a jump in oscillation amplitude associated with a switch from the $2 \mathrm{~S}$ to $2 \mathrm{P}$ vortex shedding mode (and associated jump in the phase lag between vortex force and cylinder oscillation). These observations show that vortex-induced vibration is sensitive to the detailed dynamics of vortex shedding.

In this paper we consider the case of the free vibration of circular cylinders in a tightly packed periodic square inline array of cylinders with a pitch to diameter ratio $P / D=1.5$. The role of vortex wake dynamics in the flow-induced vibration of tube arrays is still not well understood, particularly for inline arrays (whose wake dynamics are characterized by incomplete vortex shedding). Two principal theories have been advanced to explain the vibrational instability of such tube arrays: negative damping (which requires only that one tube move relative to its fixed neighbours), and stiffness controlled (which requires that adjacent tubes move out of phase in a "whirling" mode). We use appropriate numerical simulations to investigate both scenarios.

If a single cylinder free to vibrate in the transverse direction is surrounded by fixed cylinders (a common experimental configuration), the flow asymmetries caused by the movement of the central cylinder relative to its neighbours generates a "galloping" type instability in addition to the pure vortex-induced vibration of the isolated cylinder case. Although this galloping is often treated as a purely potential flow instability mechanism (except in the resonant vortex shedding case $U^{*} \approx 1 / S t$ ), the necessary forces and phase lag are determined by the interaction of the wake of the moving cylinder with the surrounding cylinders.

Price and Païdoussis (1988) developed a simple negative damping theory of fluidelastic instability in tube bundles by assuming a phase lag between the cylinder motion and total fluid force. (If there is no phase lag, the dynamics is purely potential and only a non-oscillatory divergent instability is possible.) Consider an array of cylinders as in figure 1(a) in which the central cylinder is free to vibrate in the $y$-direction transverse to the mean flow (all other 
cylinders are fixed). If the response of the cylinder is modelled as a simple harmonic oscillator, the equation of motion for the vibrating cylinder is

$$
\ddot{y}+\frac{\delta}{\pi} \omega_{N} \dot{y}+\omega_{N}^{2} y=\frac{F_{y}}{m},
$$

where $\omega_{N}$ is the natural frequency, $m$ is the mass (including added mass) and $\delta$ is the logarithmic decrement (i.e. mechanical damping). For small displacements quasi-static theory gives the total fluid force as

$$
F_{y}(t) \approx \frac{1}{2} \rho U^{2} D \frac{d C_{L}}{d y} y(t-\tau)-\frac{1}{2} \rho U D C_{D} \dot{y}(t),
$$

where the first term is the force due to the cylinder displacement and the second term is the fluid damping. $C_{L}$ and $C_{D}$ are respectively the lift and drag coefficients at equilibrium, where $C_{L}=0$ due to symmetry. Note that we assume a time lag $\tau$ between the cylinder displacement and the resulting force. This time lag leads to an additional drag term since $y(t)$ is assumed to be sinusoidal. Substituting (2) in (1), assuming that $\tau \approx \mu D / U_{g} \ll 1$ (where $U_{g}$ is mean velocity in the gap between the cylinders and $\mu \sim O(1))$ and setting the total drag equal to zero gives the critical velocity for the negative damping instability,

$$
\frac{U_{g}}{f_{N} D}=U_{\text {crit }}^{*}=\left[\frac{4}{-C_{D}-\mu D d C_{L} / d y}\right]\left(\frac{m \delta}{\rho D^{2}}\right) .
$$

This expression suggests that $d C_{L} / d y$ must be large and negative for instability. This is consistent with vortex shedding flows since a positive vortex is shed from the lower side of a cylinder when its displacement is maximal. Note that in practice the phase lag parameter $\mu$ and the linear force coefficients $C_{D}$ and $d C_{L} / d y$ must be measured experimentally.

On the other hand, if neighbouring tubes can move out of phase then tube vibration may be amplified via the stiffness-controlled mechanism. Linear stability analysis (Connors, 1978) then gives

$$
U_{\text {crit }}^{*}=C\left(\frac{m \delta}{\rho D^{2}}\right)^{1 / 2} .
$$

Observations suggest that the dominant vibrational instability is a whirling mode where adjacent tubes along a column transverse to the mean flow move in phase, while every other tube moves anti-phase: i.e. $x_{j}=x_{j+1}, y_{j}=y_{j+1}$, but $x_{j+1}=-x_{-j-1}, y_{j+1}=-y_{j-1}$. Adjacent columns of tubes move approximately anti-phase. Assuming such a whirling vibrational mode, Connors (1978) used linear stability analysis to show that the critical velocity coefficient $C$ can be expressed in terms of the linear fluid force coefficients (which can be measured experimentally).

Experimentally, it is found that if the mass damping ratio $m \delta / \rho D^{2}>0.7$ the critical velocity $U_{\text {crit }}^{*}$ follows an approximately square root dependence on the mass damping ratio as in (4). However, there is significant scatter in the measured values for $C$, with $C \approx 3.4 \pm 1.4$ for inline square arrays (Blevins, 2001). Despite their qualitative differences, equations (3) and (4) give similar results for mass damping ratios smaller than 100 provided the relevant parameters are measured experimentally. Although these expressions are based on an essentially inviscid quasi-static analysis, the effect of the vortex shedding wakes is encoded in the parameters. It is still unclear to what extent the negative damping and stiffness-controlled theories can predict flow-induced vibration, even when the relevant parameters are measured experimentally (Whiston and Thomas, 1982).

In tube arrays vortex shedding is considered to the be the primary cause of instability only when $U^{*} \approx 1 / S t$. However, vortex-induced vibration (VIV) and fluidelastic instability (FEI) are clearly not separate effects since, according to potential theory, $d C_{L} / d y>0$ and there is no phase lag between cylinder motion and the fluid force. The goal of this paper is to help elucidate the relative roles of potential flow and vortex effects in generating flow-induced vibration in tube arrays.

We focus on the following questions:

- Are the 2-D Navier-Stokes simulation results consistent with the negative damping (single degree of freedom) theory?

- Do the single moving cylinder and multiple moving cylinder cases give similarly accurate estimates of the critical flow velocity for instability? 
- What is the role of vortex-induced vibration in non-resonant fluidelastic instability?

- How accurate are periodic potential flow simulations for predicting FEI (i.e. estimating the critical velocity coefficient $C$ )?

- How accurate are 2-D periodic Navier-Stokes simulation for predicting FEI?

Since our goal is to evaluate the relative contributions of potential flow and vortex shedding to flow-induced instability, we use two distinct numerical methods: a charge simulation method for potential flow on doubly-periodic domains Amano et al. (2001) and a penalized pseudo-spectral method for the 2-D Navier-Stokes equations on periodic domains Kevlahan and Wadsley (2005). The response of the cylinder is modelled as an harmonic oscillator forced by the fluid forces (either potential alone or potential and vortical together).

\section{Method}

In order to evaluate the relative contributions of potential flow and vortex shedding to the flow-induced instability, we use two distinct numerical methods. We calculate the potential flow through the periodic tube array using a modified version of the charge simulation method Amano et al. (2001); Amaya and Sakajo (2008) . The complex potential $w(z)$ for the periodic two-dimensional flow around $J$ disks with centres $\delta_{j}$ and radii $s_{j}$ is given by

$$
w(z)=\sum_{j=1}^{J} \sum_{k=1}^{N-1} c_{k j} \log \left[\frac{\left.\theta_{1}\left(\left(z-\zeta_{k, j}\right) / L_{x}, \exp \left(-\pi L_{y} / L_{x}\right)\right)\right)}{\theta_{1}\left(\left(z-\zeta_{k+1, j}\right) / L_{x}, \exp \left(-\pi L_{y} / L_{x}\right)\right)}\right],
$$

where $c_{j k}$ are the strengths of the $N$ charges located in the interior of each of the $j=1, \ldots, J$ disks at points $\zeta_{k, j}=$ $\delta_{j}+0.7 s_{j} \exp (2 \pi \mathrm{i} k / N)$. The charge strengths are found by enforcing the no-penetration (inviscid) velocity boundary conditions at the collocation points $z_{k, j}=\delta_{j}+s_{j} \exp (2 \pi \mathrm{i} k / N)$ on the disks. The Jacobi $\theta$-function $\theta_{1}(u ; q)$ formulation ensures doubly-periodic boundary conditions, with periods $L_{x}$ and $L_{y}$ in the $x$ - and $y$-directions, respectively. Note that the cylinders can be given an instantaneous velocity by suitably modifying the boundary conditions on the disks. This method produces highly accurate results (to machine precision). Figure 3(a) shows streamlines for a typical example.

The complex force $F$ on the disk $j$ is then found by evaluating the integral

$$
F=\frac{i \rho}{2} \oint_{C_{j}}\left(\frac{d w}{d z}\right)^{2} \mathrm{~d} z
$$

which is discretized (to exponential accuracy) using the collocation points $z_{k, j}$. To the best of our knowledge, this is the first time the charge simulation method has been applied to fluid-structure interaction.

The two-dimensional vortical flow through the periodic tube array is calculated by solving the following $L^{2}$ penalized equations,

$$
\frac{\partial \boldsymbol{u}}{\partial t}+\boldsymbol{u} \cdot \nabla \boldsymbol{u}+\nabla P=\frac{1}{\operatorname{Re}} \Delta \boldsymbol{u}-\frac{1}{\eta} \chi(\boldsymbol{x}, t)\left(\boldsymbol{u}-\boldsymbol{u}_{O}\right), \quad \nabla \cdot \boldsymbol{u}=0,
$$

where the last term on the rhs of (6) approximates the no-slip boundary conditions on the surface of the cylinder moving with velocity $\boldsymbol{u}_{O}$ as $\eta \rightarrow 0$ (where the characteristic function $\chi(x, t)=1$ or 0 in the solid and fluid regions of the flow, respectively). Note that $\chi$ is the union of the characteristic functions corresponding to each cylinder $\chi_{j}(\boldsymbol{x}, t)$. The Reynolds number $R e=U_{\infty} D / v$, where $U_{\infty}$ is the mean velocity over both the solid and fluid parts of the periodic domain (equivalent to the free stream mean flow upstream of a tube array), and $D$ is the tube diameter. Note that the force on a cylinder can be found simply by integrating its penalization term,

$$
\boldsymbol{F}=\int \frac{1}{\eta} \chi_{j}(\boldsymbol{x}, t)\left(\boldsymbol{u}-\boldsymbol{u}_{O}\right) \mathrm{d} x .
$$

The penalized Navier-Stokes equations (6) are solved using a Fourier transform based pseudo-spectral method in space (e.g. Vincent and Meneguzzi, 1991) and a Krylov method in time (Edwards et al., 1994). The pseudospectral method is computationally efficient and highly accurate for spatial derivatives, while the Krylov method is 
a stiffly stable explicit method with an adaptive step-size to maintain a specified error tolerance. We consider only two-dimensional flow at a Reynolds number $R e=200$ (based on the free stream mean velocity). At this Reynolds number the flow is still approximately two-dimensional, and is characterized by strong periodic vortex shedding.

In both the potential and vortical flow simulations the response of cylinder $j$ is modelled as a forced simple harmonic oscillator,

$$
\ddot{\boldsymbol{y}}_{j}(t)+\frac{\delta}{\pi} \omega_{N} \dot{\boldsymbol{y}}_{j}(t)+\omega_{N}^{2} \boldsymbol{y}_{j}(t)=\boldsymbol{F}_{j}(t) / m,
$$

where $m$ is the mass (including added mass), $\delta$ is the logarithmic decrement (i.e. mechanical damping), $\omega_{N}$ is the natural frequency and $\boldsymbol{F}_{j}$ is the total fluid force (vortex and potential) on cylinder $j$. We assume that all cylinders are mechanically identical (i.e. $m, \delta$ and $\omega_{N}$ are the same for all cylinders). The cylinders are free either to oscillate in both directions, or only in the direction transverse to the mean flow. By coupling the potential flow force calculation to the oscillator equations (8), and introducing an artificial time lag $\tau$ in the fluid forcing, i.e. $\boldsymbol{F}_{j}(t) \rightarrow \boldsymbol{F}_{j}(t-\tau)$, one can derive a set of ordinary differential delay equations to model the negative damping instability. In the vortical flow case the cylinder motion is coupled to the fluid motion by updating the mask functions $\chi_{j}(\boldsymbol{x}, t)$ according to the coupled oscillator equations (8).

\section{Results}

\subsection{Configuration}

We investigate the flow through inline square arrays with a pitch to diameter ratio $P / D=1.5$ as shown in figure 1(a,b). The periodic unit cell contains between four and twenty-five complete cylinders (depending on the case), and either all cylinders can move in both streamwise and transverse directions (multiple degrees of freedom), or we allow only transverse motion of the central cylinder (single degree of freedom). All vortical simulations are done at Reynolds number $R e=200$.

It is important to note that there are two distinct definitions of mean velocity: the mean velocity over the entire domain (including the cylinders) $U_{\infty}$, and the velocity averaged across the gap between the tubes $U_{g} . U_{\infty}$ corresponds to the mean velocity upstream of a tube array, and measures the total mass flux through the array, while $U_{g}$ measures the typical velocity through the array. If all flow must pass through the array (i.e. for an infinite array, or for ducted arrays)

$$
U_{g}=\frac{P}{P-D} U_{\infty}=3 U_{\infty} \quad \text { if } \quad P / D=1.5 .
$$

Note that the Reynolds number based on $U_{g}$ is also larger: $R e_{g}=3 R e=600$ in our case.

As emphasized below, relation (9) does not hold for an isolated bundle of tubes. In this case some of the mean flow can by-pass the array due to the blockage effect and $U_{g}$ is correspondingly lower.

\subsection{Potential flow}

We first consider the case of potential flow, where the periodic unit cell contains $2 \times 2$ complete cylinders free to oscillate in both the transverse and streamwise directions (the transverse-only mode is stable). Since the tube array does not have a vibrational instability mode for zero phase lag (only a divergent mode), we perform a nonlinear stability analysis for a range of non-zero phase lags from 0 to 180 degrees. In order to determine the critical velocity for each phase lag we simply solve the relevant delay equations (described in \$2) for the system of coupled oscillator ordinary differential equations, with the fluid forcing for each cylinder determined by the numerical potential flow calculation. The results for the critical velocity coefficient $C$ do not depend on the mass damping ratio, at least in the range $[0.1,100]$, and are not significantly affected by using more than four cylinders in the periodic unit cell.

Figure 2 shows that the potential flow results for the critical velocity coefficient, $C \approx 5.2$ for phase lags $\phi \in$ $[40,140]$, is surprisingly close to the experimental data. Figure 5 shows that that potential flow results are within the experimental scatter for square arrays for phase lags $\phi \in[40,140]$. However, as we will see below, the actual phase lag measured in the Navier-Stokes simulations is about $168^{\circ}$, which corresponds to a significantly larger $C \approx 7.6$. This suggests that the potential flow results, although accurate, do not adequately approximate the vortical flow dynamics. In addition, the vibrational mode is strictly streamwise for the potential flow, with adjacent cylinders vibrating antiphase. This is quite different from the fully developed whirling mode seen in vortical flows where the vibration 

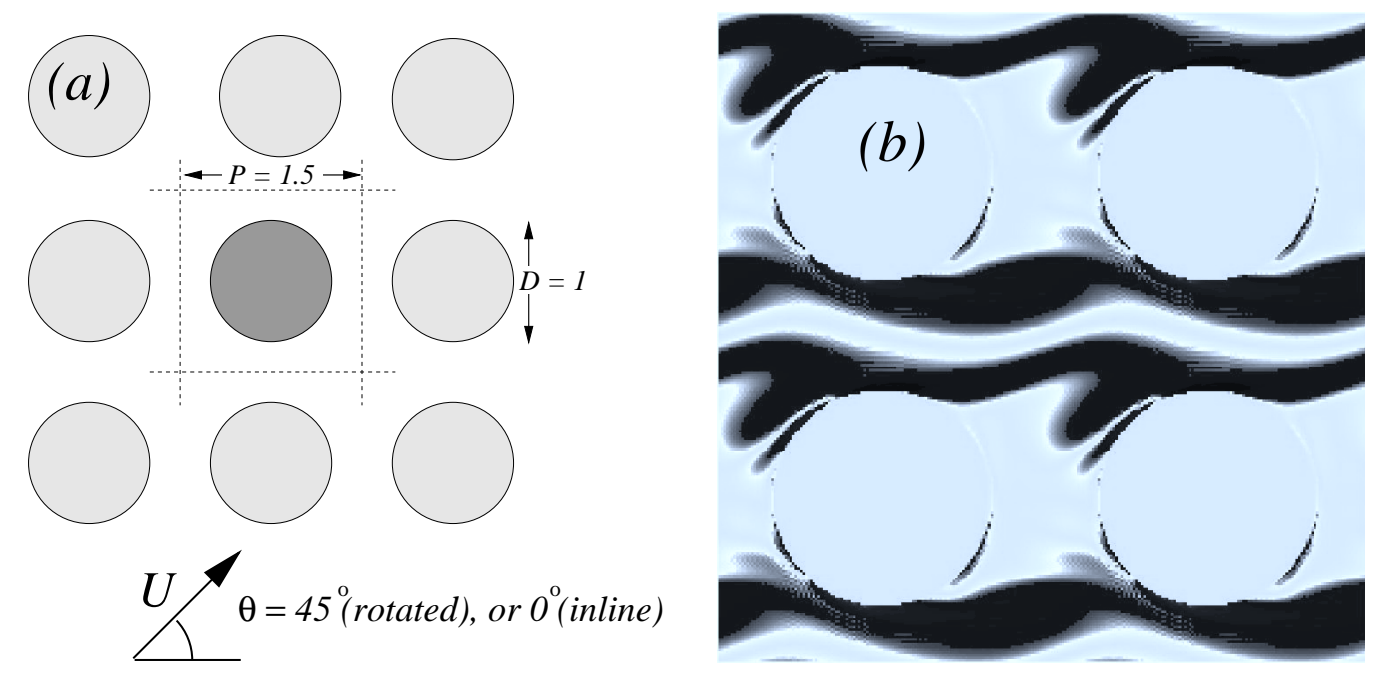

Figure 1: (a) Tube configuration for a square array of circular cylinders. We consider only inline flow. (b) Vortex wake dynamics for twodimensional flow at $R e=200$ with all cylinders fixed.

mode is essentially transverse (see 4(b)). This shows that the phase lag does not correctly model the effect of vortex shedding, even though it gives a reasonable estimate for the critical velocity.

This result appears to be inconsistent with Paidoussis et al. (1982)'s potential flow calculations, who found critical velocities three to five times higher than the experimental values. This discrepancy may be due to the fact that Paidoussis et al. (1982) apparently made their linear stability analysis using small (non-periodic) arrays assuming that the gap velocity $U_{g}=P /(P-D) U_{\infty}$. However, as mentioned earlier, this relation does not hold for isolated arrays, due to the blocking effect which diverts the mean flow around the array. In fact, this relation significantly overestimates the gap velocity for isolated arrays (by a factor of about 1.55 for a $3 \times 3$ array with $P / D=1.5$ ), which would therefore overestimate the critical velocity by a factor of about $1.55^{2} \approx 2.4$. Note that this overestimation is not improved by using larger arrays.

\subsection{Vortical flow}

We now consider numerical solutions of the full 2-D Navier-Stokes equations at $R e=200$, which allows us to directly measure the parameters in (3) and to perform nonlinear stability analyses including vortical effects. At $R e=200$ the flow is still approximately two-dimensional, and is characterized by strong periodic vortex shedding at a Strouhal frequency $S t=1.08$. In order to ensure that we are well outside the domain of the resonant vortex-induced vibration instability, we choose the natural frequency of the cylinder $f_{N}=1$, mean flow velocity $U_{\infty}=5\left(U_{g}=15\right)$, $\delta=0.1$ and mass damping ratio $m \delta /\left(\rho D^{2}\right)=1.0$. Since the Strouhal frequency $S t=1.08$ at $R e=200$, this choice of parameters ensures that we focus on the non-resonant fluidelastic regime $U^{*} \gg U_{\text {crit }}^{*} \gg 1 / S t$. We consider periodic unit cells containing between $2 \times 2$ and $5 \times 5$ cylinders (see figure 1 (a) and figure $4(\mathrm{a})$ ).

When the cylinders are fixed figure 1(b) shows that the vortex wakes do not undergo complete vortex shedding (i.e. complete detachment of the shed vortices). Nevertheless, the wake develops periodic transverse oscillations which produce a periodic oscillation of the lift force with an amplitude of 0.333 (normalized by $U_{g}$ ) and frequency 1.08 . Cylinder motion generates complete vortex shedding, similar to von Karman vortex shedding in the isolated cylinder case (see Williamson and Govardhan, 2004) and in the rotated tube array configuration (see Price et al., 1995). This observation indicates that the vibrational instability is a form of singular perturbation that qualitatively changes the wake dynamics.

When the central cylinder is free to move in the transverse direction, and all other cylinders are fixed, it undergoes large periodic oscillations. Figure 3(b) confirms that the vortex wake dynamics is strongly modified by the cylinder motion. The wake is stabilized (i.e. it becomes essentially potential flow) at the narrow gap side, while the wide gap side allows for complete vortex shedding. Comparing figures 3(b) and figure 4(a) suggests visually (as confirmed 


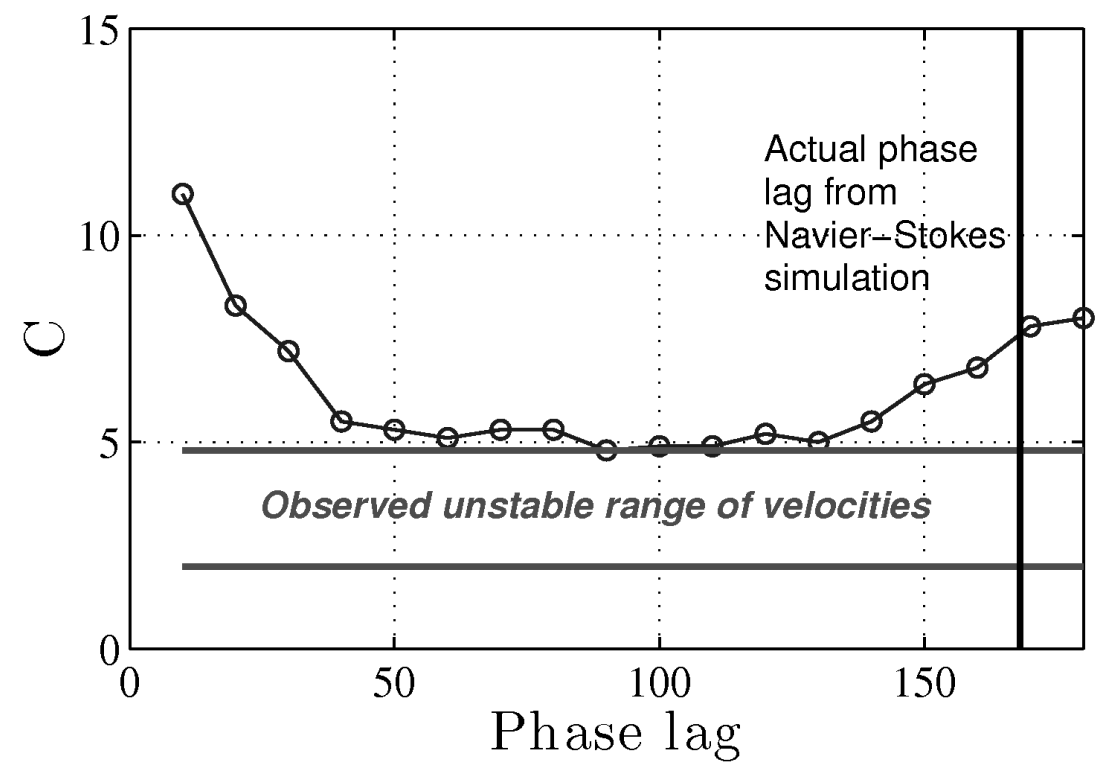

Figure 2: Nonlinear stability calculations for periodic potential flow with $2 \times 2$ unit cell. The potential flow value of the critical velocity coefficient $C$ is close to the experimental data for a wide range of phase lags.

by quantitative calculations) that the $3 \times 3$ periodic unit cell captures the main vortex wake dynamics. Figure 6 (a) shows that the vortex contribution to the total lift is large: about twice as large in magnitude as the contribution from the potential flow. However, since the contributions from the vorticity and potential flow are exactly anti-phase, the maximum amplitude of the total lift force is is similar to that of the potential flow alone. It is important to remember, however, that the potential force is proportional to cylinder displacement, and so the vortex force will strongly dominate for small amplitude vibrations. The vortex force is anti-phase with the cylinder motion (and hence the potential force) because a positive vortex is shed from the lower side of the cylinder when cylinder displacement $y(t)$ is maximum, thus generating a strong downward force. The fundamental difference between the potential flow and vortical flow is illustrated by figure 3(c), which shows that vortex shedding reverses the sign of $C_{L}(y)$, breaks monotonicity and introduces significant hysteresis $\left(C_{L}\right.$ depends on the velocity of the cylinder as well as its position). In particular, maximum lift force is achieved for intermediate (not maximum) displacements of the cylinder in the vortical flow.

It is interesting to check whether the negative damping theory (3) is consistent with the Navier-Stokes simulation results. Curve fitting shows that the cylinder displacement and lift force (normalized by the gap velocity $U_{g}=3 U_{\infty}$ ) are respectively

$$
\begin{aligned}
y(t) & =0.27 \sin 1.1 \omega_{N} t \\
C_{L}(t) & =-0.7 y(t-0.03)-0.19 \sin \left(\text { St } U_{\infty} / D 2 \pi t\right) .
\end{aligned}
$$

These results confirm that, as required for the negative damping instability mechanism (3), $d C_{L} / d y=-0.7<0$ and there is a phase lag between the fluid force and the cylinder motion of $\phi=\omega \tau=\pi-0.2$ (taking into account the change in sign). Note the distinct Strouhal frequency contribution to $C_{L}(t)$ due to vortex shedding.

However, the actual numerical results of the Navier-Stokes simulation are inconsistent with negative damping theory, even in the ideal case when only a single tube is free to vibrate transverse to the mean flow and the relevant parameters are measured precisely. Substituting the simulation values $C_{D}=0.27, \mu=\tau U_{g} / D=0.45$ and $d C_{L} / d y=$ -0.7 into (3) gives a critical velocity $U_{\text {crit }}^{*}=89$, which is much larger than the actual value $U_{\text {crit }}^{*}=8.7 \pm 0.1 \mathrm{measured}$ for the Navier-Stokes simulation, which is in turn much larger than the experimental value of about $U_{\text {crit }}^{*} \approx 3.4$. These results suggest that considering only a single (transverse) vibrational mode significantly overestimates the critical 
(a)

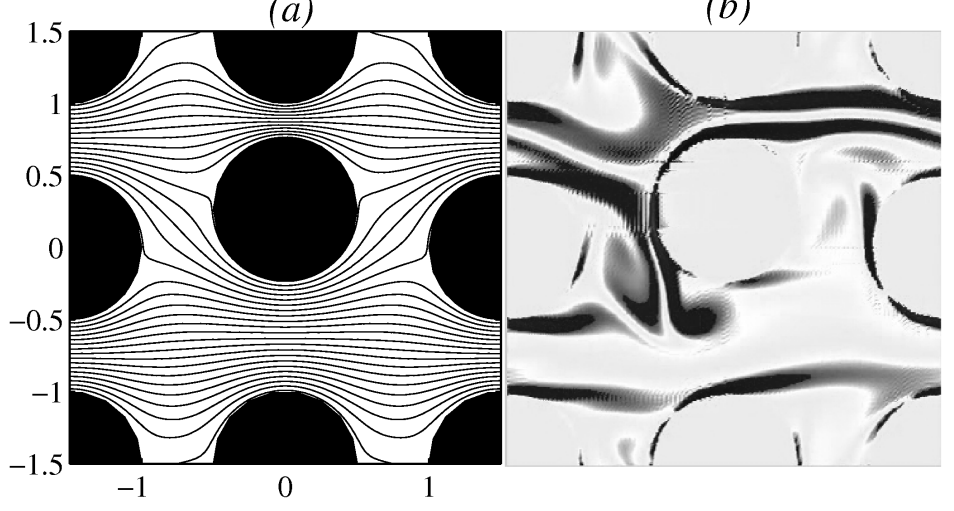

(c)

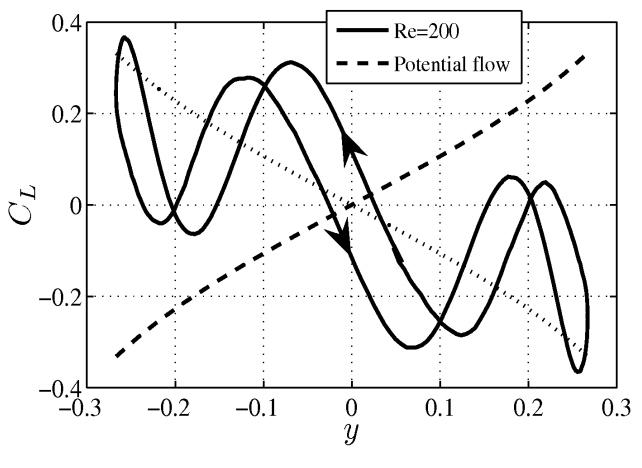

Figure 3: Flow induced vibration in an inline tube array with $P / D=1.5$. The central cylinder is free to move in the transverse direction only. (a) Potential flow streamlines. (b) $\mathrm{Re}=200$ vorticity (red is positive, blue is negative). (c) Comparison of lift force (normalized by $U_{g}$ ) as a function of cylinder position for potential flow and Navier-Stokes flow at $R e=200$. The lift force for the Navier-Stokes flow has been averaged over many periods of cylinder oscillation. The dotted line is $-C_{L}(y)$ for the potential flow and the arrows give the direction of cylinder motion (note hysteresis).

velocity. Figure 5 shows each of these results compared with the experimental data.

On the other hand, if all cylinders are free to vibrate in both directions, the 2-D Navier-Stokes simulation gives estimates for the critical velocity in good agreement with the experimental data for a wide range of mass damping ratios, as shown in figure 5. Although these results were calculated for a fixed Reynolds number, $R e=200$, they should be relatively robust as the force amplitudes do not depend sensitively on Reynolds number once vortex shedding has developed. It is interesting to note that the initial instability at onset is in fact streamwise (as in the potential flow case), although the vibration quickly switches to an essentially transverse (or, more precisely, whirling) mode, as observed in experiments. This may explain why linear stability analysis based on an anti-phase whirling mode have not successfully predicted the critical velocity in inline arrays (Whiston and Thomas, 1982).

It is instructive to separate the contributions to the fluid force from the potential and vortical parts of the flow. Figure 6(a) shows the total lift force compared with its potential and vortical parts. This decomposition is done during the Navier-Stokes simulation by first calculating the total force and then subtracting the potential force contribution calculated based on the instantaneous position and velocity of the cylinder using the charge simulation method described earlier. A curve fit shows that vortex force is almost exactly out of phase with the cylinder motion,

$$
C_{L \text { VORTex }}(t)=-2 y(t-0.01)-0.203 \sin \left(S t U_{\infty} / D 2 \pi t+0.03\right) \text {. }
$$

Because the mass damping ratio is relatively small, the amplitude of motion is large $(A=0.27)$, and the size of the potential force is also correspondingly large. Despite the relatively large potential force it is clear that the total force is controlled by its vortical component, apart from a small change in amplitude.

We now propose a physical explanation for the phase lag, and give a rough estimate for its magnitude. If we assume that the vortex force is generated by a vortex of strength $U_{g} / D R e^{1 / 2}$ shed at speed $U_{g}$ in a direction $45^{\circ}$ to the mean flow direction (where $U_{g}$ is the mean speed in the gap between cylinders), then the maximum lift force magnitude should be approximately $C_{L}$ VORTEX $=1 / \sqrt{2} R e^{1 / 2} / U_{g}$. For the case presented here, this gives $C_{L \text { VORTEX }} \approx 0.667$, in reasonable agreement with the observed value 0.541 from equation (10). (In comparison, the potential force $C_{L \text { POTENTIAL }} \approx y$ for tube arrays with $P / D=1.5$.) Note that this approximation is strictly valid only for two-dimensional flows at moderate Reynolds numbers. The phase shift may be estimated by noting that the vortex shedding begins at maximum cylinder amplitude (minimum gap). However the vortex initially moves vertically (generating no lift). The lift is maximum when the vortex moves below the cylinder into the fast downstream flow. This gives $\phi_{\text {vortex }} \approx \pi / 8 D / U_{g} \omega \approx 0.18$ in our case, in reasonable agreement with the observed value 0.2 (the $1 / 8$ of the circumference estimate was used based on observing flow animations). This estimate agrees with the experimental observation that $\tau \propto D / U_{g}$ (Blevins, 2001).

Finally, we check that the cylinder is indeed stable when forced only by the potential part of the force, even when the flow itself is vortical. Figure 6(b) shows the cylinder displacement as a function of time when forced by the total 
(a)

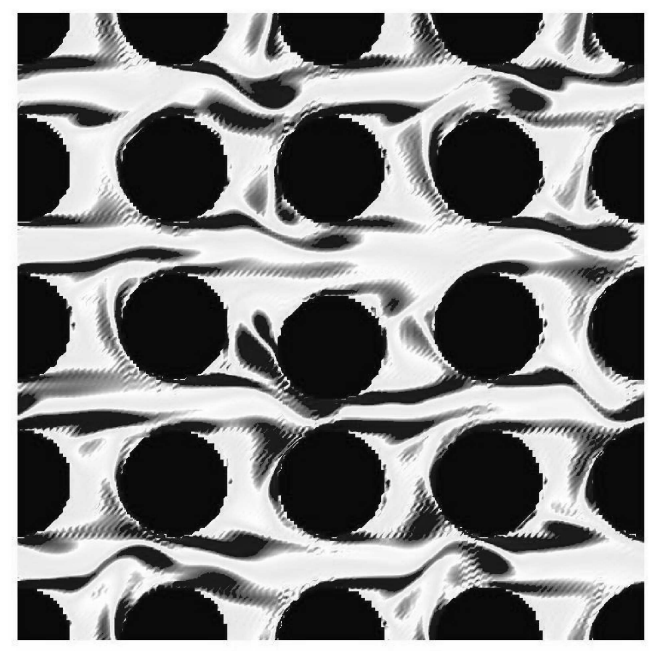

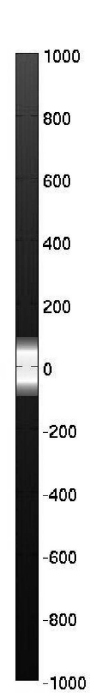

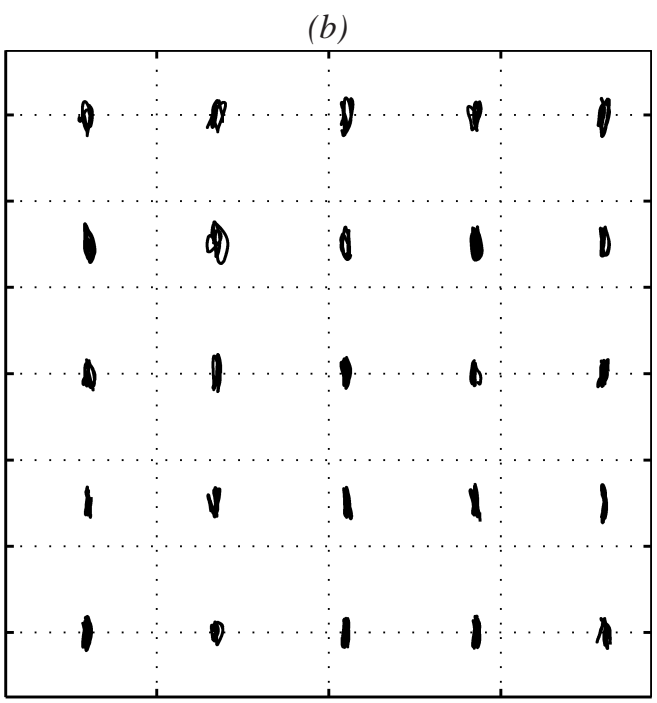

Figure 4: (a) Vorticity field at $R e=200$ for a $5 \times 5$ periodic unit cell with the central cylinder free to move in the transverse direction. (b) Cylinder trajectories for a $5 \times 5$ periodic unit cell with all cylinders free to move.

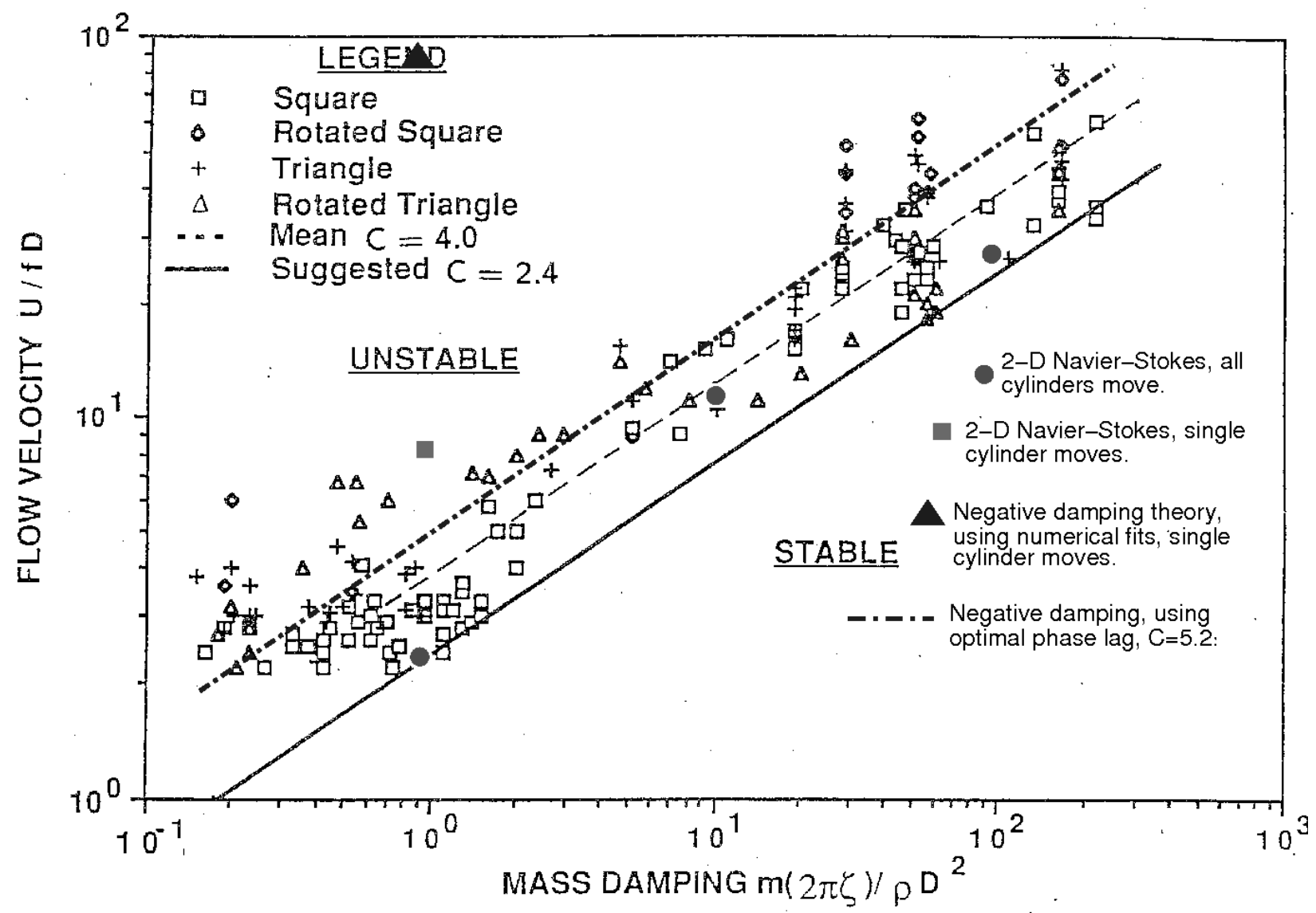

Figure 5: Potential flow and 2-D Navier-Stokes calculations compared with data and theory. Results are normalized with respect to the gap velocity $U_{g}$. (Modified from Blevins (2001) Fig. 5-6.) 
(a)

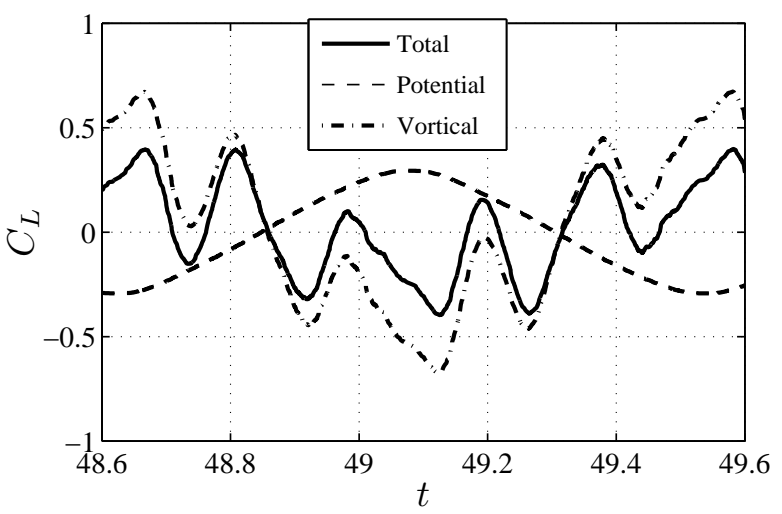

(b)

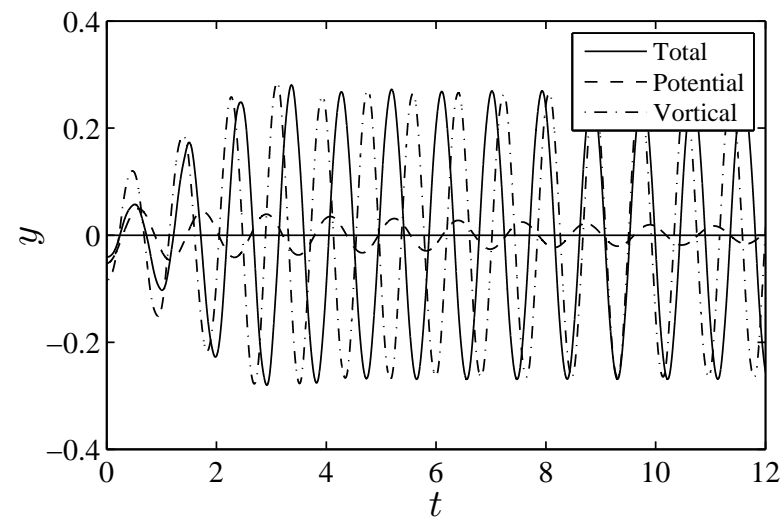

Figure 6: 2-D Navier-Stokes simulation results for a $3 \times 3$ periodic array with the central cylinder free to move in the transverse direction only and a mass damping of one. (a) Total lift compared with its potential and vortical parts. (b) Cylinder amplitude when cylinder is forced by the total force, only the potential force, or only the vortical force.

force, the potential force alone and the vortical force alone. This confirms that the overall vibrational mode is entirely due to the vortical component of the force, even though the potential force is of comparable magnitude to the total force. The crucial contribution of the vortex wake is to shift the force anti-phase to the cylinder displacement, and to introduce a new Strouhal frequency component to the force. However, it is important to remember that in this case only the central cylinder can move, and only in the transverse direction. When all cylinders can move the initial instability is potential in nature (although the fully-developed whirling instability is strongly vortical).

\section{Conclusions}

The results presented here have helped to untangle the roles of vortex shedding and potential flow in the nonresonant fluidelastic instability of tube arrays. We have shown that vortex wake dynamics (especially vortex shedding) is the dominant factor determining the vibrational stability of inline tube arrays, even in the non-resonant case.

In particular, we have shown how flow asymmetry (a potential flow effect) ensures that the vortex-generated lift force $d C_{L} / d y<0$, which is necessary for the negative damping instability mechanism. The negative damping mechanism also requires that the fluid force lag behind the cylinder motion and our results allowed us to measure this phase lag, and to understand why it is proportional to $D / U_{g}$, as observed experimentally. However, we found that the simple negative damping theory is inconsistent with the results of the 2-D Navier-Stokes simulations, even when the relevant parameters measured from the simulation are used to predict the critical flow velocity. Moreover, we found that the critical flow velocity measured in the 2-D Navier-Stokes simulations for a single cylinder moving transverse to the flow is much higher than that measured in experiments. In contrast, nonlinear stability analysis using the 2-D Navier-Stokes simulations of the case where all cylinders are free to move in both the streamwise and transverse directions gave results consistent with the experiments over a large range of mass damping ratios, from 1 to 100 .

In order to check that the fully-developed vibrational instability is controlled by the vortical part of the total fluid force (and that the potential part does not play a direct role) we performed 2-D Navier-Stokes simulations where the cylinder is forced either by the total force, or by the potential force alone, or by the vortical force alone. These numerical experiments confirmed that the vibrational instability is due to the vortical part of the force, and that the vortex shedding ensures that the force is approximately anti-phase to the cylinder motion (together with a Strouhal frequency component).

Surprisingly, we found that the potential flow simulations gave reasonably accurate predictions of the critical flow velocity for a wide range of phase lags, provided all cylinders are free to oscillate in both streamwise and transverse directions. The instability mode in this case is essentially streamwise, with adjacent cylinders moving anti-phase. We conjecture that the accuracy of the potential flow calculations is due to the fact that the initial instability mode in 
the 2-D Navier-Stokes simulations is also streamwise (although the fully-developed instability mode is the so-called whirling mode, as observed in experiments). This suggests that potential flow calculations may be far more accurate than previously thought, provided the gap velocity is measured correctly and that the cylinders are free to vibrate in the streamwise direction. It also suggests that the linear instability analysis of tube arrays should assume a streamwise anti-phase mode, rather than the usual whirling (or transverse) anti-phsase mode.

In summary, we found that 2-D Navier-Stokes simulations of periodic arrays of cylinders give good predictions of the critical flow velocity for the vibrational instability of inline tube arrays over a wide range of mass damping ratios. The fact that the potential flow stability analysis also gives reasonably good results for the critical flow velocity is surprising and requires further analysis, focusing on the form of the initial instability mode. The initial instability mode is likely to be qualitatively different from the fully-developed mode because the dynamics of the wake changes qualitatively in inline arrays once the tubes start to vibrate (from jet-like to von Karman-like with complete vortex shedding).

\section{References}

Amano, K., Okano, D., Ogata, H., Shimohira, H., Sugihara, M., 2001. A systematic scheme of numerical conformal mapping of unbounded multiply-connected domains by the charge simulation method. Information Processing Society of Japan Journal 43 (3), $385-395$.

Amaya, Y., Sakajo, T., 2008. Charge simulation method for approximating the complex potential in a channel domain with multiple circular islands. In: JST Presto Symposium on Mathematical Sciences towards Environmental Problems (Hokkaido University technical report series in mathematics ; 136). pp. 42-45.

Blevins, R., 2001. Flow-induced vibration, 2nd Edition. Krieger Publishing Company.

Connors, H., 1978. Fluidelastic vibration of heat exchanger tube arrays. Journal of Mechanical Design 100, $347-353$.

Edwards, W. S., Tuckerman, L. S., Friesner, R. A., Sorensen, D. C., 1994. Krylov methods for the incompressible Navier-Stokes equations. Journal of Computational Physics 110, 82-102.

Kevlahan, N. K.-R., Wadsley, J., 2005. Suppression of three-dimensional flow instabilities in tube bundles. Journal of Fluids and Structures 20 (4), 611-620.

Paidoussis, M., Mavriplis, D., Price, S., 1982. A potential-flow theory for the dynamics of cylinder arrays in cross-flow. Journal of Fluid Mechanics 146, 227-252.

Païdoussis, M. P., Price, S.J. 1988. The mechanisms underlying flow-induced instabilities of cylinder arrays in cross-flow. Journal of Fluids Mechanics $187,45-59$

Price, S. J., Païdoussis, M. P., Mark, B., 1995. Flow visualization of the interstitial cross-flow through parallel trangular and rotated square arrays of cylinders. Journal of Sound and Vibration 181, 85-98.

Vincent, A., Meneguzzi, M., 1991. The spatial structure and statistical properties of homogeneous turbulence. Journal of Fluid Mechanics 225, $1-20$.

Whiston, G., Thomas, G., 1982. Whirling instabilities in heat exchanger tube arrays. Journal of Sound and Vibration 81 (1).

Williamson, C., Govardhan, R., 2004. Vortex-induced vibration. Annual Review of Fluid Mechanics 36, $413-455$. 


\section{Figure captions}

Figure 1: (a) Tube configuration for a square array of circular cylinders. We consider only inline flow. (b) Vortex wake dynamics for two-dimensional flow at $R e=200$ with all cylinders fixed.

Figure 2: Nonlinear stability calculations for periodic potential flow with $2 \times 2$ unit cell. The potential flow value of the critical velocity coefficient $C$ is close to the experimental data for a wide range of phase lags.

Figure 3: Flow induced vibration in an inline tube array with $P / D=1.5$. The central cylinder is free to move in the transverse direction only. (a) Potential flow streamlines. (b) $\mathrm{Re}=200$ vorticity (red is positive, blue is negative). (c) Comparison of lift force (normalized by $U_{g}$ ) as a function of cylinder position for potential flow and NavierStokes flow at $R e=200$. The lift force for the Navier-Stokes flow has been averaged over many periods of cylinder oscillation. The dotted line is $-C_{L}(y)$ for the potential flow and the arrows give the direction of cylinder motion (note hysteresis).

Figure 4: (a) Vorticity field at $R e=200$ for a $5 \times 5$ periodic unit cell with the central cylinder free to move in the transverse direction. (b) Cylinder trajectories for a $5 \times 5$ periodic unit cell with all cylinders free to move.

Figure 5: Potential flow and 2-D Navier-Stokes calculations compared with data and theory. Results are normalized with respect to the gap velocity $U_{g}$. (Modified from Blevins (2001) Fig. 5-6.) Figure 6: 2-D Navier-Stokes simulation results for a $3 \times 3$ periodic array with the central cylinder free to move in the transverse direction only and a mass damping of one. (a) Total lift compared with its potential and vortical parts. (b) Cylinder amplitude when cylinder is forced by the total force, only the potential force, or only the vortical force. 